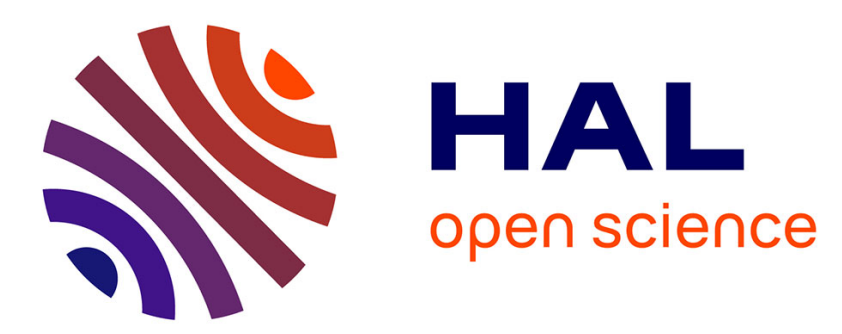

\title{
Ultrasound scattering from cell pellet biophantoms can provide insight into the cellular structure involved in scattering \\ Pauline Muleki Seya, William O’Brien
}

\section{- To cite this version:}

Pauline Muleki Seya, William O'Brien. Ultrasound scattering from cell pellet biophantoms can provide insight into the cellular structure involved in scattering. 2020 IEEE International Ultrasonics Symposium (IUS), Sep 2020, Las Vegas, United States. pp.1-4, 10.1109/IUS46767.2020.9251710 . hal-03110511

\author{
HAL Id: hal-03110511 \\ https://hal.science/hal-03110511
}

Submitted on 18 Oct 2021

HAL is a multi-disciplinary open access archive for the deposit and dissemination of scientific research documents, whether they are published or not. The documents may come from teaching and research institutions in France or abroad, or from public or private research centers.
L'archive ouverte pluridisciplinaire HAL, est destinée au dépôt et à la diffusion de documents scientifiques de niveau recherche, publiés ou non, émanant des établissements d'enseignement et de recherche français ou étrangers, des laboratoires publics ou privés. 


\section{Ultrasound scattering from cell pellet biophantoms can provide insight into the cellular structure involved in scattering}

\author{
Pauline Muleki-Seya \\ Université de Lyon, INSA Lyon, UCBL \\ CREATIS \\ 69100 Villeurbanne, France \\ pauline.muleki-seya@creatis.insa-lyon.fr
}

\author{
William D. O'Brien, Jr. \\ ECE Department, University of Illinois \\ Bioacoustics Research Laboratory \\ 61801 Urbana, USA \\ wdo@uiuc.edu
}

\begin{abstract}
The cellular structure involved in ultrasound scattering is still not identified. Indeed, it is not always easy to correlate the ultrasound parameters estimated through quantitative ultrasound techniques with cellular structures from histology. The use of an ultrasound scattering model adapted for concentrated media, the structure factor model (SFM), allowed us previously to estimate scatterer parameters close to cellular structures for ex vivo tissue, suggesting information about the cellular structure involved in ultrasound scattering. In this study, ultrasound scatterer parameters from 4T1 cell pellet biophantoms were estimated with two ultrasound scattering models: the spherical Gaussian model (GM) and the SFM to obtain an insight about the scattering from nuclei only and cells only. Then, numerical scatterer parameters were estimated for scattering from cells, nuclei, and both cells and nuclei using the mean nucleus and cell radii and volume fractions of $4 \mathrm{~T} 1$ from histology. The comparison between GM and SFM suggests a contribution of coherent and incoherent BSC and that the medium may be considered as concentrated. The comparison between the scatterer parameters (radii, volume fractions) from experimental and numerical distributions suggests a scattering from both cells and nuclei of $4 \mathrm{~T} 1$ cell pellet biophantoms.

Index Terms - Quantitative ultrasound techniques, Ultrasound scattering, cell pellet biophantoms, Cellular structure.
\end{abstract}

\section{Introduction}

Quantitative ultrasound techniques provide information about tissue microstructure through the evaluation of the backscatter coefficient (BSC). Scatterer parameters are extracted by fitting the measured BSC with a theoretical BSC evaluated using an ultrasound scattering model. However, scatterer parameters are often difficult to correlate to specific tissue microstructure(s) involved in ultrasound scattering at the cellular scale. The difficulty may come from the use of inappropriate ultrasound scattering models, such as a diluted media model (i.e. spherical Gaussian Model), used classically, and taking only the contribution of the incoherent BSC. In previous work, we showed that the use of an ultrasound scattering model adapted for concentrated media (structure factor model), combining the contributions of the incoherent

NIH Support: R37EB002641. and coherent BSC, provided scatterer parameters close to cellular structures: nuclei for canine liver and whole cells for HT29 tumors [1]. At cellular scale, this study suggested that the nuclei may be the main source of scattering from the canine liver and that whole cells may be the main source of scattering from HT29 murine tumors. The ultrasound scattering structure at the cellular scale is still not identified. Other studies, based on the numerical study of rabbit liver 3-D impedance maps [2] or the effects of apoptosis [3], [4], [5] suggested that the nuclei are the dominant source of scattering at high frequency.

The goal of this paper is to obtain an insight into the cellular structure(s) involved in ultrasound scattering. We assume that in case of scattering from nuclei only, the BSC will be composed of an incoherent contribution only and that the GM should provide reasonably accurate estimates of the nuclei. In the case of scattering from cells only, the BSC will be composed of incoherent and coherent contributions, and that the SFM should provide reasonably accurate estimates of the cells. To determine if both cells and nuclei are involved in ultrasound scattering, numerical scatterer parameters using mean nuclei and cells radii and volume fractions of $4 \mathrm{~T} 1$ cell pellet biophantoms will be estimated and compared to the experimental outcomes.

\section{Material and Methods}

\section{A. Ultrasound acquisition}

The cell pellet biophantoms were composed of densely packed cells without any supportive background materials. 4T1 mammary carcinoma cells (ATCC \#CRL-2539) were used to create the biophantoms $(\mathrm{n}=16)$. The experimental procedure to make these biophantoms was described in [6].

Ultrasound acquisitions were realized with a singleelement, weakly focused transducer centered around 40-MHz (High Frequency Transducer Resource Center, USC, Los Angeles, CA, $-10 \mathrm{~dB}$ bandwidth of $25-55 \mathrm{MHz}$ ). 
The scanning procedure, described in [6], was composed of the acquisition of the radio-frequency (RF) signals and attenuation measurements (insertion-loss broadband technique [7]). For each sample, 11 independent scans were recorded. A mean BSC was estimated for each of the 11 scans by averaging the BSCs from all the ROIs within that scan. Then, for each cell pellet biophantom sample, 11 mean BSCs were extracted and used to estimate the scatterer parameters.

\section{B. Parameter evaluation}

1) Parameters estimated from histological images: Immediately after scanning, the sample was prepared for histology processing. Cell nuclei and radii and their volume fractions were estimated from histological images of these biophantoms. Assuming that the nuclei and cells had circular shapes, the corresponding nuclei and cell radii were computed from the area estimates using ImageJ on at least 150 nuclei. A procedure was then performed to approximately obtain the nuclei and cell volume fractions from the 2-D histological images using the number of nuclei in the image and the mean nuclei/cell radii as well as cell diameter for the thickness dimension.

2) Quantitative Ultrasound (QUS) parameters: This paper compared QUS estimates from two models: the spherical Gaussian model (GM) and the Structure Factor Model (SFM). Using the GM, the BSC was modeled using a spatial autocorrelation function describing the size, shape, acoustic properties, and distribution of the scatterers in the medium. The BSC was expressed as the product of the BSC in the Rayleigh limit and the backscatter form factor FF [8]. The form factor describes the frequency dependence of the scattering in terms of the size, shape, and acoustic properties of the scatterers. The Gaussian form factor models a medium with continuous changes in acoustic properties. The hypotheses of this model make it valid only for diluted media. The theoretical BSC using the SGM formulation is given by [8]:

$$
B S C_{G M}(k)=\frac{k^{4} V_{s}^{2} \eta_{Z}}{4 \pi^{2}} e^{-0.827 k^{2} a_{G}^{2}} ;
$$

where $k$ denotes the wavenumber, $\eta_{Z}$ the acoustic concentration, $a_{G}$ the effective scatterer radius and $V_{s}=4 / 3 \pi a_{G}^{3}$. The unknown parameters are the scatterer radius $a_{G}$ and the acoustic concentration $\eta_{Z}$.

The SFM is based on the assumption that, at high scatterer volume fractions, interference effects are mainly caused by correlations between the spatial positions of individual scatterers (discrete scatterer with impedance differing from that of a homogeneous background medium). By considering an ensemble of identical spheres of radius $a$, the theoretical BSC for the SFM formulation is given by [9], [10]:

$$
B S C_{S F M}(k)=n \frac{k^{4} V_{s}^{2} \gamma_{Z}^{2}}{4 \pi^{2}}\left[\frac{3}{(2 k a)^{3}} j_{1}(2 k a)\right]^{2} S(k) ;
$$

where $V_{s}$ is the sphere volume and $n=\phi / V_{s}$ their number density with $\phi$ the scatterer volume fraction, $\gamma_{Z}$ is the relative impedance contrast between the scatterer and the surrounding medium and $j_{1}$ is the spherical Bessel function of the first kind of order $1 . S$ is the structure factor, which can be analytically obtained as described in [[10], eqs. (A1)-(A4)] based on [11]. The unknown parameters are the scatterer radius $a$, the volume fraction $\phi$, and the relative impedance contrast $\gamma_{Z}$. The acoustic concentration for the SFM was also calculated as: $\eta_{Z}=\phi \gamma_{Z}^{2} /\left(4 / 3 \pi a^{3}\right)$.

Estimated values of the QUS parameters were determined by fitting the measured $B S C_{\text {meas }}$ to the theoretical BSCs, $B S C_{\text {theo }}$, by minimizing the cost function:

$$
F=\frac{\sum_{j}\left\|B S C_{\text {meas }}\left(k_{j}\right)-B S C_{\text {theo }}\left(k_{j}\right)\right\|^{2}}{\sum_{j} B S C_{\text {meas }}\left(k_{j}\right)^{2}}
$$

where $B S C_{\text {theo }}$ is given by eq. 2 . The cost functions were minimized over 25-55 MHz. The fitting procedure was performed using the minimization routine fminsearch without constraint in MATLAB (The MathWorks, Inc., Natick, MA). The best cost function was evaluated using 20 initial conditions.

3) Numerical scatterer parameters: Theoretical BSCs for scattering from nuclei only, $B S C_{N}$, cells only, $B S C_{C}$, and both nuclei and cells, $B S C_{N C}$, were evaluated as:

$$
\begin{array}{r}
B S C_{N}(k)=B S C_{S F M}\left(k, a_{N}, \phi_{N}\right) \\
B S C_{C}(k)=B S C_{S F M}\left(k, a_{C}, \phi_{C}\right) \\
B S C_{N C}(k)=r B S C_{N}(k)+(1-r) B S C_{C}(k)
\end{array}
$$

where, $a_{N}, \phi_{N}, a_{C}, \phi_{C}$ denote the radius and volume fraction from nucleus and cell, respectively. $r$ is between 0 and 1 and describes the ratio of scattering from nuclei and cells: a ratio of 0 corresponds to scattering from cells only and a ratio of 1 corresponds to scattering from nuclei only.

A relative impedance contrast, $\gamma_{Z}$, of 0.03 was used to generate $B S C_{N}$ and $B S C_{C}$. This value was chosen when comparing theoretical BSCs from nucleus and cell with the measured BSCs for different values of $\gamma_{Z}$ (Fig. 1A.). An example of theoretical BSCs for scattering from cells only, nuclei only, and both cells and nuclei (for different values of $r$ ) are presented in Fig. 1B using mean nucleus and cell parameters of $4 \mathrm{~T} 1$ from histology.

In order to extract different sets of numerical scatterer parameters, $81 B S C_{N}$ (respectively $B S C_{C}$ ) were 
generated using 81 random $a_{N}$ and $\phi_{N}$ (respectively $a_{C}$ and $\phi_{C}$ ) values with $a_{N}$ (respectively $a_{C}$ ) comprised between $\left[\operatorname{mean}\left(a_{N}\right)-\operatorname{std}\left(a_{N}\right), \operatorname{mean}\left(a_{N}\right)+\operatorname{std}\left(a_{N}\right)\right]$ (respectively $\left.\left[\operatorname{mean}\left(a_{C}\right)-\operatorname{std}\left(a_{C}\right), \operatorname{mean}\left(a_{C}\right)+\operatorname{std}\left(a_{C}\right)\right]\right)$ and $\phi_{N}$ (respectively $\left.\phi_{C}\right)$ comprised between $\left[\operatorname{mean}\left(\phi_{N}\right)-\right.$ 0.25 mean $\left.\left(\phi_{N}\right), \operatorname{mean}\left(\phi_{N}\right)+0.25 \operatorname{mean}\left(\phi_{N}\right)\right]$ (respectively $\left.\left[\operatorname{mean}\left(\phi_{C}\right)-0.10 \operatorname{mean}\left(\phi_{C}\right), \operatorname{mean}\left(\phi_{C}\right)+0.10 \operatorname{mean}\left(\phi_{C}\right)\right]\right)$ from 4T1 histology measurements. The ratio $r$ used were 0 (cells only), $0.1,0.2,0.3,0.4,0.5,0.6,0.7,0.8,0.9$ and 1 (nuclei only). Eighty-one $B S C_{N C}$ were generated for each ratio using the corresponding $B S C_{N}$ and $B S C_{C}$. The same process, as used experimentally, was realized to estimate the numerical scatterer parameters.
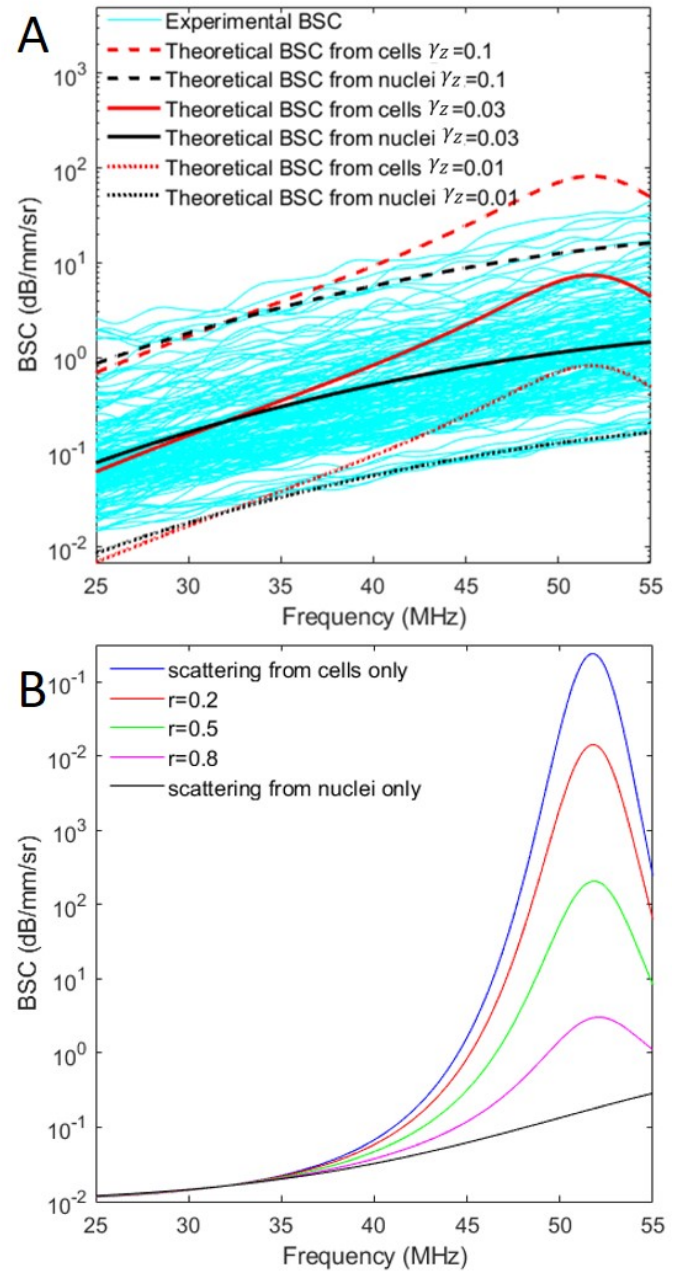

Fig. 1. A. Experimental mean BSCs obtained for $4 \mathrm{~T} 1$ and theoretical BSCs for scattering from nuclei only and cells only (with nucleus/cell radii and volume fractions estimated from histology) for three values of relative impedance contrast $\gamma_{Z}(0.1,0.03$ and 0.01$)$. Note that this figure is on a log scale only for the sake of representation. B. Example of BSCs for scattering from cells only, both cells and nuclei and nuclei only for the mean nucleus/cell radii and volume fractions estimated from histology.
TABLE I

Ultrasound scatterers parameters $\left(a, \phi\right.$ and $\left.\eta_{Z}\right)$ estimated with the Gaussian model (GM) and structure factor model (SFM) and the mean nuclei and cells radii and their volume fractions from histology for $4 \mathrm{~T} 1$ cell pellet biophantoms.

\begin{tabular}{c|ccc}
\hline & \multicolumn{3}{|c}{ Scatterer parameters } \\
& $a(\mu \mathrm{m})$ & $\phi$ & $\eta_{Z}\left(\mathrm{~dB} / \mathrm{mm}^{3}\right)$ \\
\hline US SFM & $5.86 \pm 2.14$ & $0.48 \pm 0.33$ & $30.49 \pm 14.22$ \\
US GM & $1.35 \pm 1.24$ & & $52.56 \pm 16.25$ \\
nucleus & $5.35 \pm 0.56$ & 0.14 & \\
cell & $8.12 \pm 0.68$ & 0.49 & \\
\hline
\end{tabular}

\section{Results and Discussion}

\section{A. Quantitative Ultrasound parameters}

The scatterer parameters estimated with SFM and GM are summarized in Table 1 along with nucleus and cell parameters from histology. The scatterer parameters estimated with GM present lower scatterer radii (close to $1 \mu \mathrm{m}$ ) and higher acoustic concentration than with SFM. These radii are not corresponding to any cellular structure. These results suggest that these media may be considered as concentrated (with the contribution of incoherent and coherent BSC), illustrated with an underestimation of the scatterer radius with an overestimation of the acoustic concentration with classical models [12]. It may suggest that cells or both nuclei and cells are involved in ultrasound scattering for $4 \mathrm{~T} 1$ cell pellets. The SFM scatterer parameters provide a lower relative error with the nucleus radius $(\sim 9.5 \%)$ but a lower relative error with cell volume fraction $(\sim$ $2 \%)$. The SFM scatterer parameters radius vs volume fraction present a large dispersion (Fig. 2A): 18\% of scatterers with radii and volume fractions much lower than nuclei values (section 3 ), $30 \%$ of scatterers with radii and volume fractions between those from nuclei and cells (section 5) and $45 \%$ of scatterers with volume fractions much higher than cells volume fractions (sections 7, 4 and 1). The numbered sections used in this description are presented in Fig. 2B.

\section{B. Numerical scatterer parameters}

To determine if the scatterer parameters $(a, \phi)$ representations may result from scattering from nuclei, cells, or both cell and nucleus, numerical scatterer parameters using nuclei and cells radii and volumes fractions from 4T1 were evaluated. These numerical $(a, \phi)$ representations are presented in Fig. 2B-F. Scattering from cells only leads to numerical $(a, \phi)$ representations close to cell radii and volume fractions (Fig. 2B). Scattering from both cells and nuclei results in scatterers with radii and volume fractions much lower than nuclei values (section 3), scatterers with radii and volume fractions between those from nuclei and cells (section 5) and with volume fractions much higher than cells volume fractions (sections 4, 7 and 1) (Fig. 2C-E). Scattering from nuclei only results in 

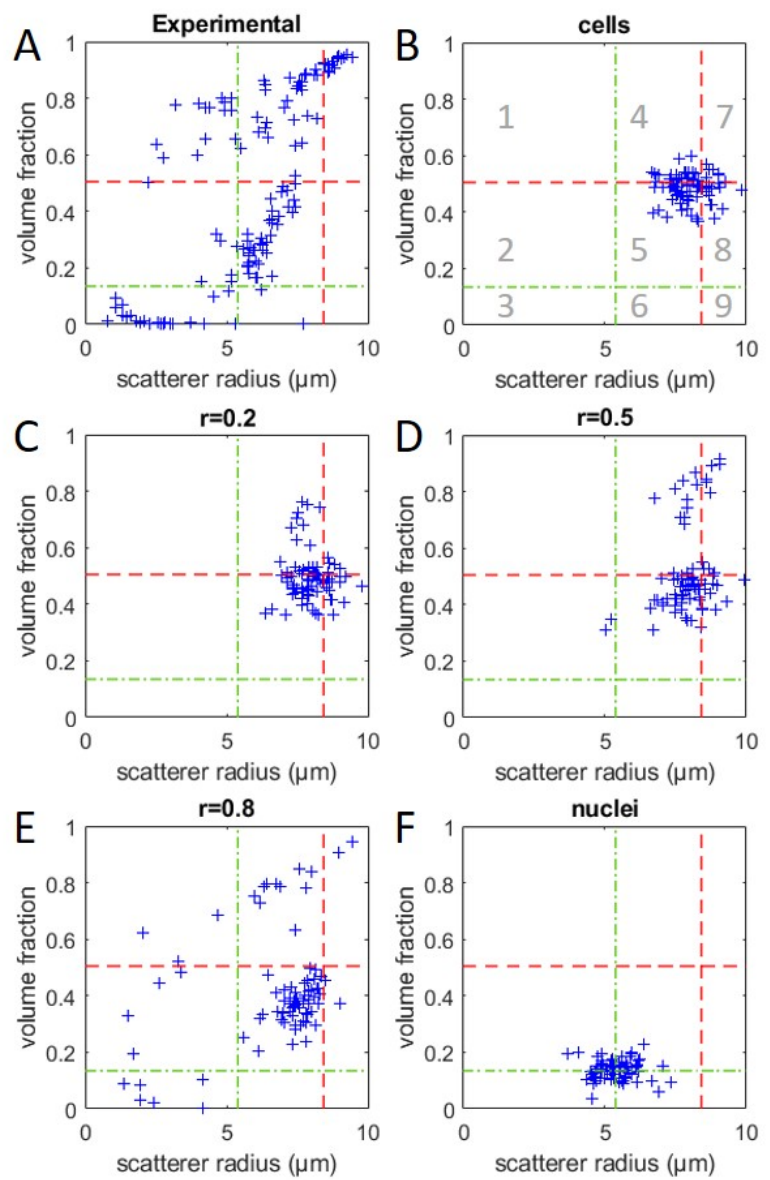

Fig. 2. Numerical ultrasound scatterer parameters radius $(a)$ vs volume fraction $(\phi)$ from $4 \mathrm{~T} 1$ experimental BSC and 4T1 numerical $\mathrm{BSC}$ for a scattering from cells only, both cells and nuclei and nuclei only. The green dot and dashed and red dashed lines correspond to the mean nuclei and cells radii and volume fractions from histology. The numbers in $\mathrm{B}$ denotes the different sections.

scatterers close to nuclei values (Fig. 2F). The percentage of scatterers present in each section for scattering from cells only, nuclei only and both cells and nuclei were extracted and their correlation with the experimental $(a, \phi)$ distribution highlighted that the best correlation is with a scattering from both cells and nuclei with a ratio $r=0.9$ $(R=0.78)$. These results suggest that both cells and nuclei are involved in ultrasound scattering from $4 \mathrm{~T} 1$ cell pellet biophantoms.

\section{Conclusion}

The objective of this study was to obtain a better insight into the cellular structure(s) involved in ultrasound scattering. For that, the first step consisted to evaluate and compare the scatterer parameters from GM and SFM with those from histology. In the case of scattering from nuclei only, pertinent scatterer parameters were expected from GM with the contribution of only incoherent BSC. In the case of scattering from cells only, pertinent scatterer parameters were expected from SFM with the contribution of coherent and incoherent BSC. Then numerical scatterer parameters were evaluated from scattering from cells only, nuclei only, and both cells and nuclei. The scatterer parameters (radii, volume fractions) distributions were compared to the experimental outcomes. This method, applied to $4 \mathrm{~T} 1$ cell pellet biophantoms, suggests that both cells and nuclei are involved in ultrasound scattering in this case.

\section{Acknowledgment}

This research was supported by the NIH grant (R37EB002641). We thank Jamie Kelly and Jake Berndt for their help in ultrasound acquisitions.

\section{References}

[1] P. Muleki-Seya, R. Guillermin, J. Guglielmi, J. Chen, T. Pourcher, E. Konofagou, and E. Franceschini, "HighFrequency Quantitative Ultrasound Spectroscopy of Excised Canine Livers and Mouse Tumors Using the Structure Factor Model," IEEE Trans. Ultrason., Ferroelectr., Freq. Control, vol. 63, no. 9, pp. 1335-1350, 2016.

[2] A. D. Pawlicki, A. J. Dapore, S. Sarwate, and W. D. O'Brien, "Three-dimensional impedance map analysis of rabbit liver," J. Acoust. Soc. Am., vol. 130, no. 5, pp. EL334-EL338, 2011.

[3] J. W. Hunt, A. E. Worthington, and A. T. Kerr, "The subtleties of ultrasound images of an ensemble of cells: simulation from regular and more random distributions of scatterers," Ultrasound Med. Biol., vol. 21, no. 3, pp. 329-341, 1995.

[4] G. J. Czarnota, M. C. Kolios, H. Vaziri, S. Benchimol, F. P. Ottensmeyer, M. D. Sherar, and J. W. Hunt, "Ultrasonic biomicroscopy of viable, dead and apoptotic cells," Ultrasound Med. Biol., vol. 23, no. 6, pp. 961-965, 1997.

[5] G. J. Czarnota, M. C. Kolios, J. Abraham, M. Portnoy, F. P. Ottensmeyer, J. W. Hunt, and M. D. Sherar, "Ultrasound imaging of apoptosis: high-resolution non-invasive monitoring of programmed cell death in vitro, in situ and in vivo," Br. J. Cancer, vol. 81, no. 3, pp. 520-527, 1999.

[6] A. Han, R. Abuhabsah, R. J. Miller, S. Sarwate, and W. D. O'Brien, "The measurement of ultrasound backscattering from cell pellet biophantoms and tumors ex vivo," J. Acoust. Soc. Am., vol. 134, no. 1, pp. 686-693, 2013.

[7] K. A. Wear, T. A. Stiles, G. R. Frank, E. L. Madsen, F. Cheng, E. J. Feleppa, C. S. Hall, B. S. Kim, P. Lee, W. D. O. Brien, M. L. Oelze, B. I. Raju, K. K. Shung, T. A. Wilson, and J. R. Yuan, "Interlaboratory Comparison of Ultrasonic Backscatter Coefficient Measurements From 2 to $9 \mathrm{MHz}$," J. Ultrasound Med., vol. 24, no. 9, pp. 1235-1250, 2005.

[8] M. F. Insana, D. G. Brown, K. K. Shung, et al., "Acoustic scattering theory applied to soft biological tissues," Ultrasonic scattering in biological tissues, pp. 75-124, 1993.

[9] V. Twersky, "Low-frequency scattering by correlated distributions of randomly oriented particles," J. Acoust. Soc. Am., vol. 81, no. 5, pp. 1609-1618, 1987.

[10] E. Franceschini and R. Guillermin, "Experimental assessment of four ultrasound scattering models for characterizing concentrated tissue-mimicking phantoms," J. Acoust. Soc. Am., vol. 132, no. 6, pp. 3735-3747, 2012.

[11] M. S. Wertheim, "Exact Solution of the Percus-Yevick Integral Equation for Hard Spheres," Phys. Rev. Lett., vol. 10, no. 8, pp. 321-323, 1963.

[12] E. Franceschini, R. d. Monchy, and J. Mamou, "Quantitative Characterization of Tissue Microstructure in Concentrated Cell Pellet Biophantoms Based on the Structure Factor Model," IEEE Trans. Ultrason., Ferroelectr., Freq. Control, vol. 63, no. 9, pp. 1321-1334, 2016. 